\title{
OS ASPECTOS SOCIOAMBIENTAIS NO ESTADO DO MARANHÃO EM TEMPOS DE PANDEMIA
}

\section{THE SOCIO-ENVIRONMENTAL ASPECTS IN MARANHÃO STATE IN TIMES OF PANDEMIC}

\author{
Aichely Rodrigues da Silva \\ Doutora em Geografia, Universidade Federal do Maranhão - Campus Grajaú e \\ SEDUC Imperatriz - Maranhão \\ aichely@hotmail.com
}

Paulo Henrique Schlickmann Doutor em Geografia, Colégio Bom Jesus e Secretaria Municipal de Ensino de Itajaí - Santa Catarina phschlickmann@gmail.com

Dailson Coelho Abreu
Mestre em Biologia de Agentes Infecciosos e Parasitários, Instituto Federal do Maranhão - Açailândia
dailson@ifma.edu.br

\section{RESUMO}

A relação direta entre a saúde humana e a qualidade do ambiente refletem os aspectos sociais de determinada população, especialmente em tempos de pandemia. A pesquisa teve o objetivo de relacionar casos confirmados de Covid-19 nos municípios maranhenses aos aspectos socioeconômicos, cobertura do saneamento e saúde preventiva. A pesquisa analisou os municípios do Estado do Maranhão, para isso foram elaborados mapas temáticos das variáveis e realizada a análise de correlação espacial pelo Índice de Moran (Global e LISA). Esta pesquisa demonstrou que nas regiões Norte, Centro e Leste do Maranhão exibiram os piores indicadores socioeconômicos e a maior concentração de casos confirmados de Covid-19. Em relação aos aspectos socioeconômicos, notou-se que a desigualdade social, da falta de coleta e tratamento de efluentes domésticos e o baixo Índice de Desenvolvimento Humano municipal (IDHm) são potenciais difusores da doença. Além disso, também há a correlação positiva entre os dados de piores índices socioeconômicos e de saúde preventiva no Estado do Maranhão. Essa análise e contribuirá com a aplicação de políticas públicas quanto à saúde e à relação com a qualidade ambiental e socioeconômica nas regiões mais vulneráveis do Estado do Maranhão.

Palavras-chave: Covid-19. Qualidade Ambiental. Socioeconômico. Maranhão.

\begin{abstract}
The direct relationship between human health and environmental quality reflect the social aspects of a given population, especially in pandemic times. The research aimed to relate confirmed cases from Covid-19 in Maranhão counties to socioeconomic aspects, sanitation coverage and preventive health. The research analyzed the municipalities of the State of Maranhão. The research analyzed the municipalities of the State of Maranhão. For that purpose, thematic maps of the variables were prepared and the spatial correlation analysis was performed using the Moran Index (Global and LISA). This research showed that in the Northern, Central and Eastern regions of Maranhão exhibited the worst socioeconomic indicators and the highest concentration of confirmed cases of Covid-19.In relation to the socioeconomic aspects, it is noted that social inequality, lack of collection and treatment of domestic sewage and low Municipal Human Development Index are potential diffusers of the disease. In addition, there is also a positive correlation between the data from the lowest socioeconomic index and preventive health in the state of Maranhão. This analysis will contribute to the application of public policies regarding health and the relationship with environmental and socioeconomic quality in the most vulnerable regions of the State of Maranhão.
\end{abstract}

Keywords: Covid-19. Environmental Quality. Socioeconomic. Maranhão.

Recebido em: 08/09/2020

Aceito para publicação em: 10/11/2020 


\section{INTRODUÇÃO}

A problemática socioambiental é caracterizada pelos conflitos entre desenvolvimento econômico e a preservação ambiental (FERNANDES e SAMPAIO, 2008). Neste sentido, em tempos de pandemia nota-se a relação direta entre a saúde humana e a qualidade do ambiente, tais fatores refletem os aspectos sociais de determinada população (RIGOTTO e AUGUSTO, 2007). A interface saúde e aspectos os socioeconômicos nos ajuda a compreender as fragilidades que a população e o meio ambientes estão submetidos, ao passo que também possibilita a visualização de oportunidades de investimentos ou áreas sub investidas. A promoção da saúde ocorre por meio de um complexo processo condicionado por diferentes fatores, incluindo o ambiente (FRIESTINO; BALDISSERA; SANTOS, 2020).

A aproximação entre a Geografia e a Epidemiologia, segundo Junqueira (2009) teve as discussões iniciadas como Hipocrátes, o pai da medicina, ao analisar a distribuição regional das doenças, a partir, da aplicação dos recursos cartográficos. O mapeamento das epidemias foi dado por John Snow (1854) devido à epidemia de cólera no centro de Londres, que provocou 14.600 mortes. Snow realizou esse estudo cartográfico da cólera que deu grande contribuição do que seria posteriormente denominada geografia aplicada (BARCELLOS, BUZAl; HANDSCHUMACHER, 2018).

No campo da geografia a análise holística dos aspectos socioambientais em determinado local é essencial para a compressão da vulnerabilidade social e ambiental da população como no atual tempo de pandemia, causada pela Covid-19. A geografia da saúde permite concatenar às questões atuais relacionadas aos fenômenos de emergências patológicas, à difusão de hospedeiros e agentes infecciosos ou à produção de desigualdades em saúde (BARCELLOS, BUZAl; HANDSCHUMACHER, 2018).

Para a análise de correlação de fatores como saúde e aspectos socioambientais são utilizados Sistemas de Informações Geográfica (SIGs). Essa técnica é utilizada para a vigilância e monitoramento de doenças transmitidas por vetores doenças transmissíveis, na saúde ambiental, dentre outras (CLARKE, MCLAFFERTY, TEMPALSKI, 1996). O SIG contribui para a política e o planejamento, e a distribuição espacial, principalmente de doenças infectocontagiosas e parasitárias (GUIMARÃES, 2016).

A relação de ambiente e saúde no Brasil resulta direta e indiretamente das políticas públicas, econômicas e sociais e não pode ser considerada independentemente dessas políticas (PELICIONE, 1998). A relação entre casos confirmados de Covid-19 e aspectos socioeconômicos no Estado do Maranhão deverá demostrar a interligação entre saúde ambiental e a saúde humana.

Para Lana et al. (2020) a epidemia de SARS-CoV-2, causador da doença COVID-19, no final de 2019 em Wuhan, na China. Todavia, outra epidemia já tinha surgido como a SARS que emergiu em Hong Kong (China), em 2003, com letalidade de aproximadamente $10 \%$ e a síndrome respiratória do Oriente Médio (MERS) na Arábia Saudita em 2012 com letalidade de cerca de 30\%. Ambos fazem parte da lista de doenças prioritárias para pesquisa e desenvolvimento no contexto de emergência.

Este manuscrito tem como objetivo relacionar dados confirmados de Covid-19 nos municípios maranhenses e aspectos socioeconômicos. A pesquisa está estruturada em: a) abordagem da qualidade das águas e a relação de casos de Covid-19; b) correlação de fatores socioeconômicos e os casos confirmados de Covid-19; por fim, as considerações finais com base no enfoque teóricometodológico e nos resultados obtidos.

\section{METODOLOGIA}

\section{Área de Estudo}

O Estado do Maranhão possui o maior potencial hídrico dos estados nordestinos, apresentando rios perenes, bom índice de chuvas, rede lacustre e um extenso litoral (RIOS, 2005). O território maranhense apresenta clima tropical e equatorial; relevo composto de planícies de aplainamento a planícies fluviomarinhas, baixos platôs e chapadas (CPRM, 2013); e a vegetação predominante é de cerrado e floresta amazônica. Os municípios maranhenses estão representados na figura 1 por nome e número. 
Figura 1 - Localização dos municípios maranhenses por nome e número.

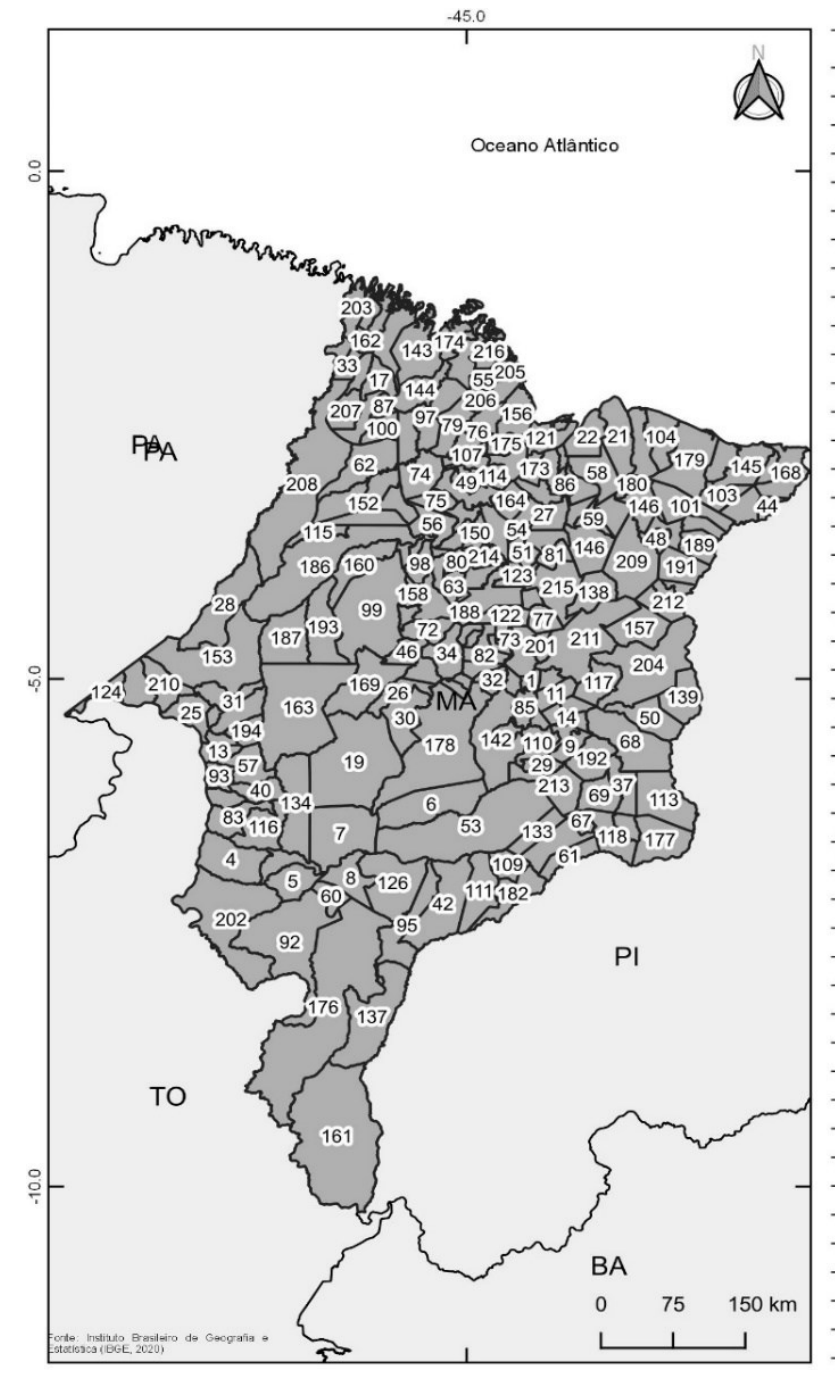

DOl:http://dx.doi.org/10.14393/Hygeia16057180
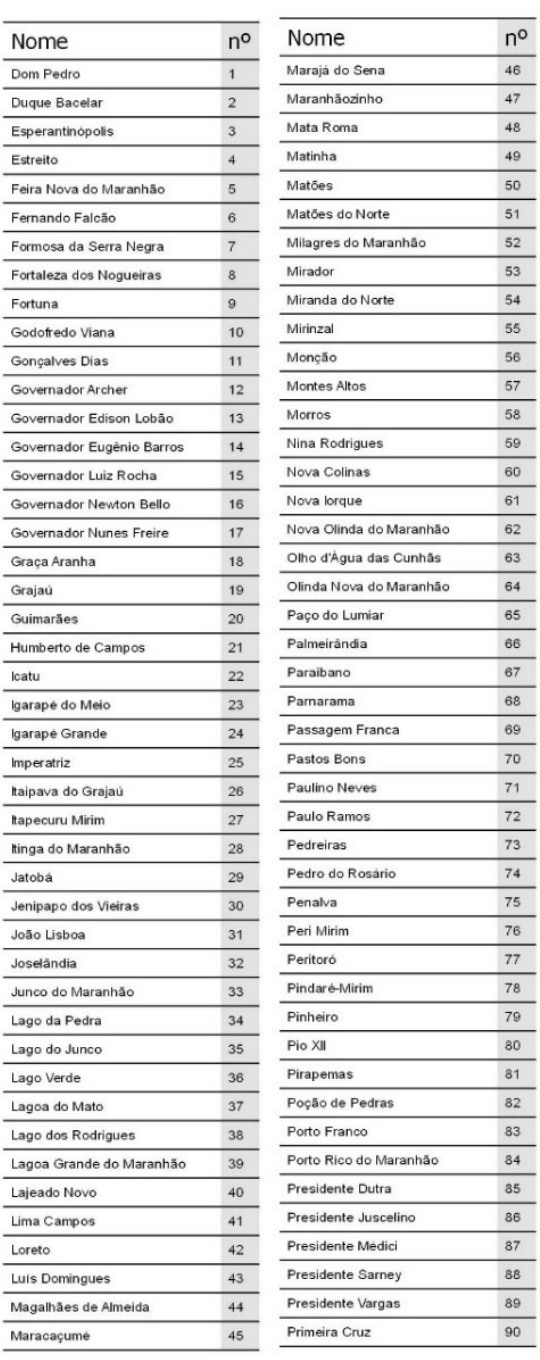

Fonte: Autores (2020)

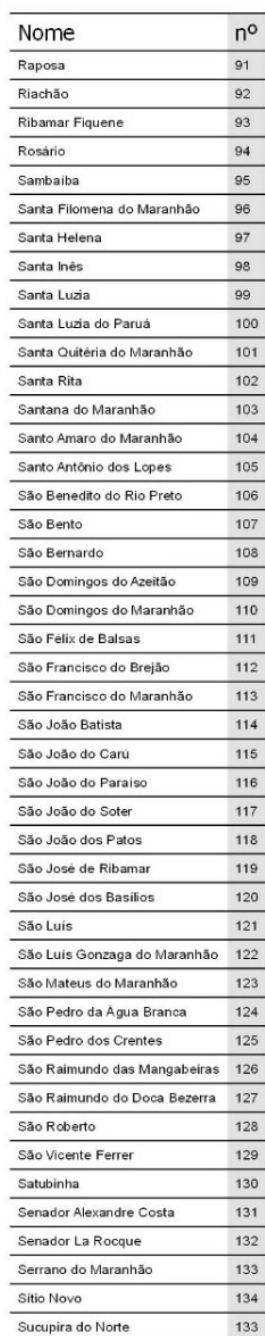

$n^{\circ}$ Nome Sucupira do Riachso

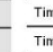

Triatelat do valt

$\frac{1+1}{1+2}$

$\frac{143}{\text { Turiagu }} \frac{14}{\text { Turitandia }}$

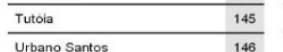

Vargem Grande 140

Via Nova dos Martice

Vhoria do mearie

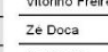

Acolathas

Atonso Cunha

Aqua Doce do Maranhas

Actantara

Altanitra do Maranhiso

Alto Alegereso Pinater

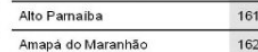

Amarante do Maranhao

Anagatoba

Anspous

Aproum:aq

\begin{tabular}{ll}
167 \\
\hline Araiones
\end{tabular}

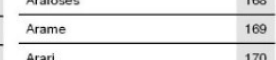

Arat

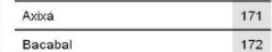

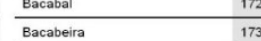

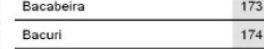

.

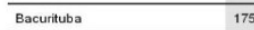

\begin{tabular}{lll}
\hline Balsas & 176 \\
\hline
\end{tabular}

Barăo de Grajau

${ }_{34}{ }^{33}$ Barra do Cord

${ }_{134} \frac{\text { Barreirinhas }}{\text { Belagua }}$
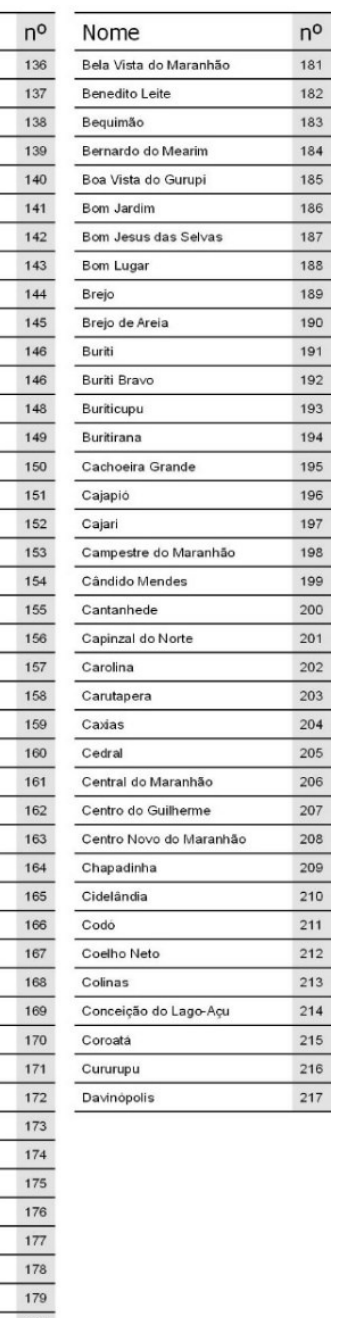

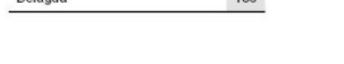

Hygeia

v.16 p. $419-430, \quad 2020$ página 421 
A área do território maranhense é de $331.937 \mathrm{~km}^{2}$, e a população estimada de 7.075 .181 habitantes e densidade demográfica de 19,8 hab/km² (IBGE, 2019). O rendimento médio do maranhense foi de $\mathrm{R} \$$ 636,00 , conforme o IBGE. Outros dados socioeconômicos como Índice de Desenvolvimento Humano (IDH), foi de 0,639 , considerado baixo. A esperança de vida ao nascer estimada é de cerca de 71 anos, sendo uma das menores do país. Neste sentido, a taxa de analfabetismo da população apresentava o maior percentual do país, em 2019, ou seja, $16,6 \%$ da população do estado com 25 anos ou mais de idade, segundo dados da Pesquisa Nacional por Amostra de Domicílios Contínua, desenvolvida pelo IBGE (2019).

\section{Estimador de Kernel (Mapa de Calor)}

Nesta pesquisa os casos confirmados de Covid-19 foram representados pelo Kernel. Para Almeida (2017) esse estimador é uma técnica exploratória que serve para analisar o comportamento de pontos e estimar a intensidade pontual do processo em toda área de estudo. O estimador de densidade kernel desenha uma vizinhança circular ao redor de cada ponto da amostra, correspondendo ao raio de influência, e então é aplicada uma função matemática de 1, na posição do ponto, a 0 , na fronteira da vizinhança (SOUZA et al., 2013). O valor para a célula é a soma dos valores kernel sobrepostos, e divididos pela área de cada raio de pesquisa. É um método muito útil e de fácil interpretação para o conhecimento da distribuição de eventos de primeira ordem.

\section{Índice de Moran (Global e Lisa)}

A análise exploratória de dados geoespaciais associados a feições de área foi baseada no Índice de Moran. O índice de Moran Global indica que -1 o agrupamento de valores dissimilares; 0 não é autocorrelação; +1 indica agrupamento de valores semelhantes. A análise local das variáveis foi dada pelo Moran Local Indicators of Spatial Association (LISA), com a aplicação do k-neighbord (vizinhos mais próximos) que é um método não paramétrico de análise. Os padrões de distribuição dos indicadores foram examinados em menor escala por meio do Moran local (LISA), produzindo um valor específico para cada município, permitindo a visualização de agrupamentos de municípios com valores similares para os indicadores selecionados.

Nessa correlação local, os tipos "Alto-Alto" demostra elevados casos confirmados de Covid-19 e elevado indicador (saneamento, fatores socioeconômico e percentual de médicos por habitantes); seguido de "Baixo-Baixo", "Alto-Baixo" e "Baixo-Alto", nos mapas temático estão apenas as análises com nível de significância $p \leq 0,05$. Para a aplicação dessas análises foi utilizado o software GeoDA e o QGIS. A análise exploratória pelo índice de Moran Global e LISA se deu como o proposto por Nunes (2013) e Luzardo, Castañeda Filho e Rubim (2017).

\section{Temática em análise}

As variáveis foram descritas nos mapas temáticos, utilizando o método corocromáticos apresentam dados geográficos e utilizam diferenças de cor na implantação zonal (ARCHELA e THÉRY,2008) com auxílio do software QGIS 2.18 Las Palmas. Para compreender a temática foram utilizados alguns indicadores que estão descritos abaixo.

\section{Qualidade e quantidade da água e saneamento básico}

Os dados relacionando a qualidade dos corpos hídricos e de demanda bioquímica de oxigênio (DBO), que corresponde à quantidade de oxigênio consumido na degradação da matéria orgânica no meio aquático por processos biológicos, sendo expresso em miligramas por litro (mg/L). Esses dados foram disponibilizados pela Agência Nacional das Águas (ANA) e os dados do Índice de Segurança Hídrica no Plano Nacional de Segurança Hídrica. Os dados de saneamento foram retirados do Atlas do Esgoto.

\section{Socioeconômico}

Os dados referentes ao Produto Interno Bruto (PIB) per capita foram disponibilizados pelo Instituto Brasileiro de Geografia e Estatística. Além disso, foram utilizados o Índice de Desenvolvimento Humano (IDH), proposto em 1947, dentro do Programa das Nações Unidas para o Desenvolvimento (PNUD). O PNUD estabelece três níveis de qualidade para o desenvolvimento humano: IDH baixo, para valores entre 0 e 0,5; IDH médio, para valores entre 0,5 e 0,8 ; IDH alto, para valores entre 0,8 e 1,0. Ademais, o Gini também foi aplicado, que é uma medida estatística de desigualdade, muito usada para indicar o grau de concentração de renda de uma região. 


\section{Saúde}

Os casos confirmados de Covid-19 (Sars-Cov-2) foram disponibilizados pela secretária Estadual de Saúde do Maranhão dados do dia 31 de julho, período que a quantidade de casos apresentou queda no Estado. Além disso, foram analisados o número de médicos por habitante, conforme dados do IBGE (2019).

\section{RESULTADOS E DISCUSSÕES}

\section{A água e a Covid-19}

O Estado do Maranhão apresentou casos elevados de Covid-19, como demostrado na figura 2A pela concentração de casos (MARANHÃO, 2020). A maior concentração desses casos ocorreu na parte Centro e Norte do estado. Com destaque para São Luís, capital do estado, como mais de 16 mil casos confirmados e 1.138 óbitos; e o município de Imperatriz, segundo maior do estado, com mais de 4 mil casos confirmados e 226 óbitos, na data de coleta dos dados para esta pesquisa.

Figura 2 - Casos confirmados de Covid-19 e qualidade da água dos corpos hídricos (A) e Índice de Segurança Hídrica e qualidade das águas dos corpos hídricos (B).

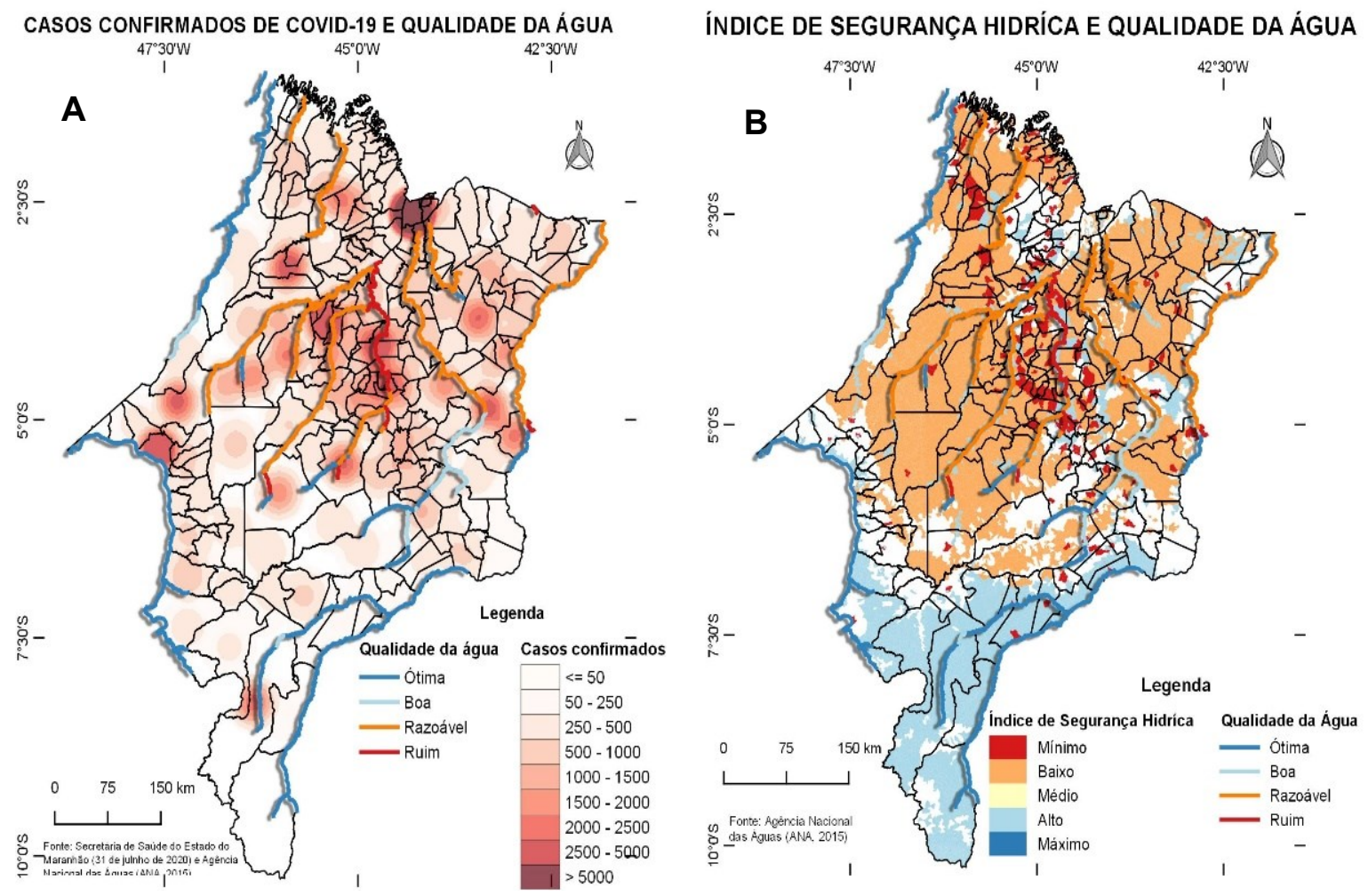

Fonte: Autores (2020).

Essas áreas também foram as que apresentaram a qualidade da água entre razoável e ruim. $\mathrm{A}$ qualidade da água e a falta de saneamento básico está relacionada a proliferação de doenças de veiculação hídrica. No Maranhão em 2018 foram internadas 42.188 pessoas com doenças de veiculação hídrica, sendo 91 óbitos (TRATABRASIL, 2020). Para Silva e Fonseca (2018) os dados de qualidade da água ainda são incipientes e Santos e Leal (2013) destacou que os instrumentos de gerenciamento dos recursos hídricos estão no estágio inicial. 
Por conseguinte, a falta de gerenciamento desses cursos d'água, indica que a água poderá ser um vetor de doenças que são transmitidas pela falta de higiene pessoal e doméstica, deficiência no abastecimento, contato com a água contaminada, vetores aquáticos (animais e pescados) e disseminação de água contaminada. Conforme a Organização Mundial de Saúde (2020) embora seja possível a presença do vírus da COVID-19 na água de beber não tratada, ele ainda não foi detectado nessas fontes de água. Conforme a Fundação Nacional da Saúde (FUNASA, 2010) as doenças relacionadas ao saneamento ambiental inadequado são: doenças de transmissão feco-oral (diarreia, febre entérica, hepatite A), doenças transmitidas por inseto vetor (dengue, febre amarela, leishmaniose, filariose linfática, malária e doença de chagas), doenças transmitidas através do contato com a água (esquistossomose e leptospirose), doenças relacionadas com a higiene (doenças dos olhos, tracoma, conjutivites, doenças de pele e micoses superfícies), geo-helmintos e teníase (helmintíases) e teníase.

A qualidade ruim das águas dos corpos hídricos se deu pelo fato que conforme o relatório da Trata Brasil (PAINEL DO SANEAMENTO, 2020), no Maranhão $86,2 \%$ da população maranhense não tem coleta e tratamento de esgoto. As soluções individuais (fossa) foram utilizadas por $15 \%$ da população. Esse dado é preocupante, pois, Casanova et al. (2009) alertou para um possível surto de SARS, que ressurgiria devido à água contaminada com os resíduos fecais de indivíduos infectados podem ser um veículo para transmissão. Ademais, não podemos ignorar a presença de outros possíveis contaminantes presentes nas águas sem tratamento adequado, como: nanopartículas, fármacos, pesticidas, produtos de higiene, cosméticos, nanoplásticos, etc., que podem estar interagindo entre si, criando condições de elevada toxicidade ou mesmo de favorecimento de manutenção e diversificação de vírus (ZHOU et al. 2003).

Outro fator preocupante no Maranhão foi a segurança hídrica, considerada baixa em grande parte do território maranhense, na figura $2 \mathrm{~B}$. A segurança hídrica é dada pela quantidade e qualidade suficientes para atender as necessidades humanas e econômicas e à conservação dos ecossistemas aquáticos, com um nível aceitável de risco relacionado a secas e cheias. Para Melo e Johnsson (2017), em tempos de mudanças ambientais globais, o conceito de "segurança hídrica" tem surgido como forma de orientar a gestão dos recursos hídricos. Esses autores, destacaram ainda que a segurança hídrica alerta para a disponibilidade de água para os usos múltiplos que atenda às expectativas da sociedade, além de protegê-la contra os efeitos negativos dos eventos hidrológicos extremos.

$\mathrm{Na}$ figura $3.1 \mathrm{~A}$, demostrou que $73 \%$ (163) dos municípios maranhenses têm de 80 a $100 \%$ da população sem coleta e tratamento de esgotos. Ao aplicar o Moran Global entre casos confirmados e coleta e tratamento de esgoto nos municípios o resultado foi de $-0,03$, com variáveis dissimilares. Para o índice LISA, a classificação Alta-Alta foi encontrada em nove municípios, ou seja, foram elevados o número de casos de Covid-19 e de percentual da população sem coleta e tratamento de esgotos (Figura 3.1B). A precária condição ambiental dos rios brasileiros e a exclusão hídrica deixa milhares de pessoas sem acesso à água de qualidade e causa impacto aos ecossistemas exigindo mudanças efetivas de atitude em relação à água e à vida.

A falta de coleta e tratamento de esgoto foi constatada na concentração de DBO nos corpos hídricos são maiores nos maiores municípios, como São Luís, Imperatriz, Açailândia e Balsas, que apresentaram 16.055, 4.594 e 2.817 casos confirmados, nesta ordem. Dentre os municípios analisados vinte exibiram mais de $1.000 \mathrm{Kg} \mathrm{DBO} / \mathrm{dia}$, sobretudo, na capital, na qual as concentrações desse indicador foram cerca de $17 \mathrm{mil} \mathrm{kg} / \mathrm{DBO} / \mathrm{dia}$. A falta de saneamento básico na llha de São Luís, foi demonstrada por Silva et al., (2014) na pesquisa que detectou alto estado trófico e más condições de para a vida aquática do Rio Anil e afluentes. Para Barcelos e Quitério (2006) a análise da saúde ambiental é considerada um fator indispensável para o controle social, para definir as necessidades e subsidiar a formulação de políticas públicas. 
Figura 3 - Indicadores (A) e análise de correlação com os casos de Covid-19 (B)
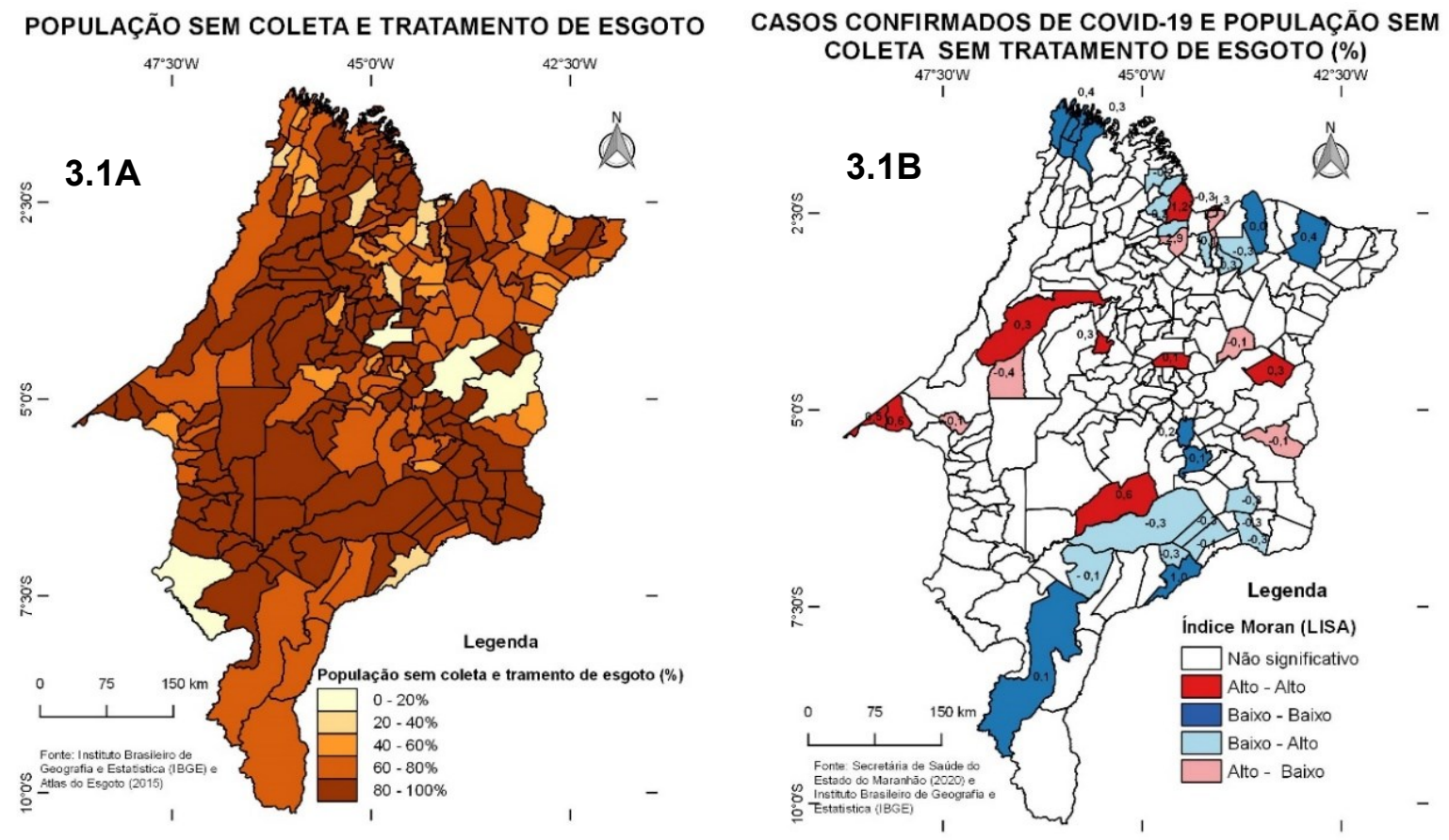

Fonte: Autores (2020). 

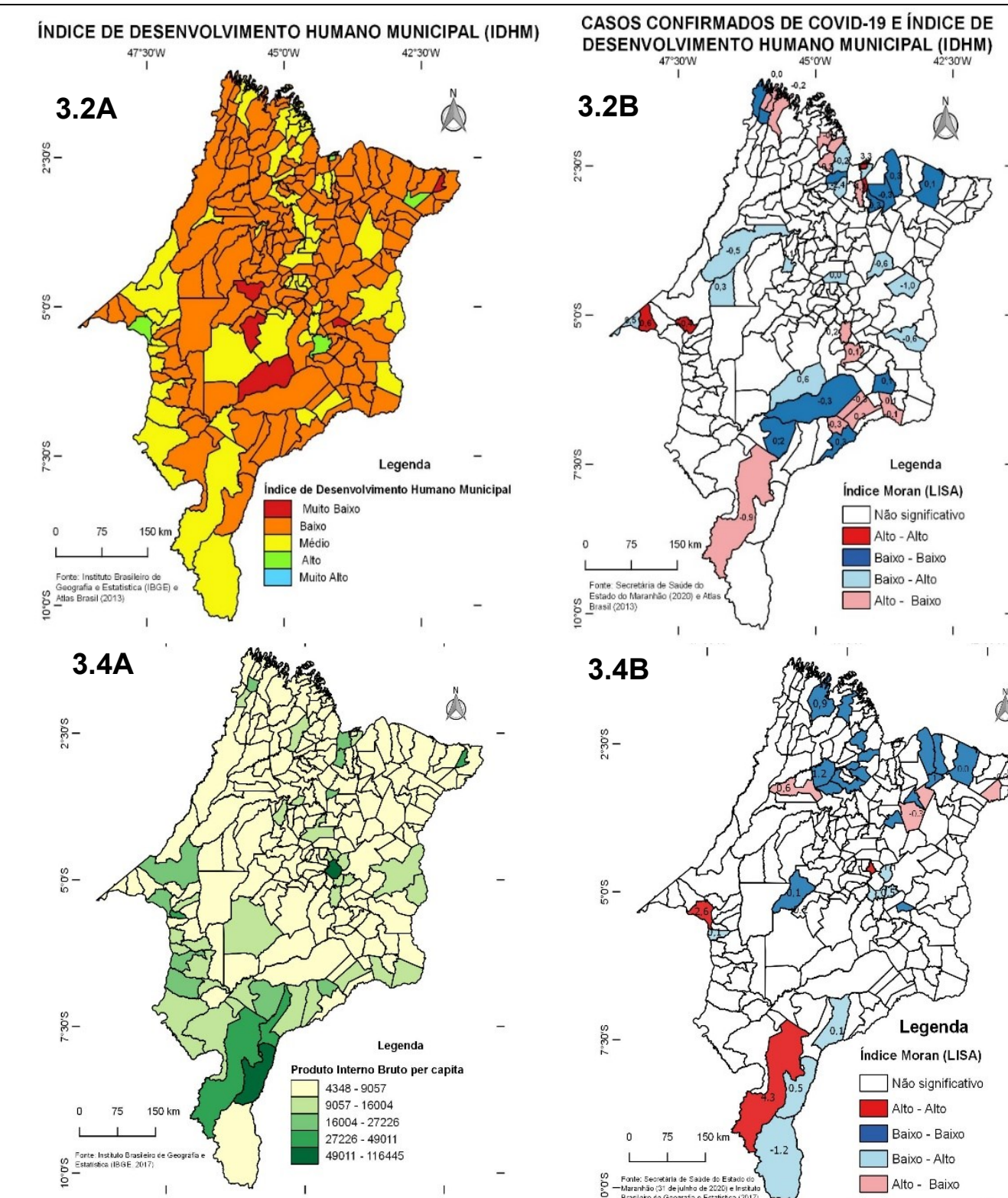

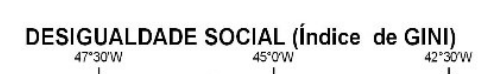

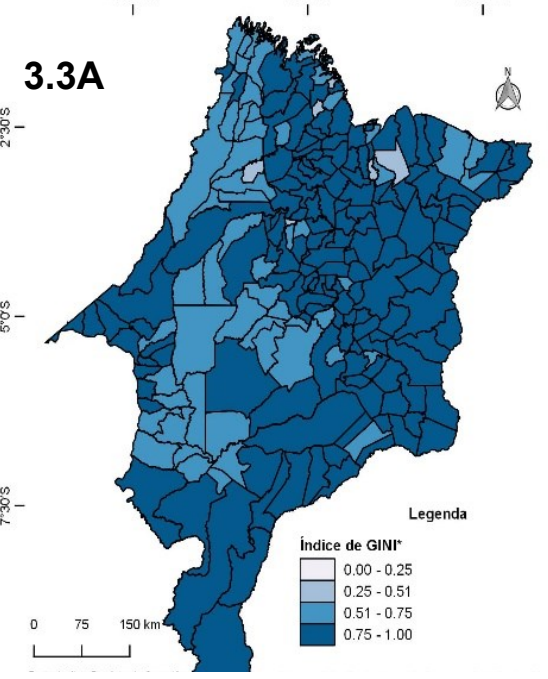

RELAÇÃO DE MÉDICOS POR HABITANTE

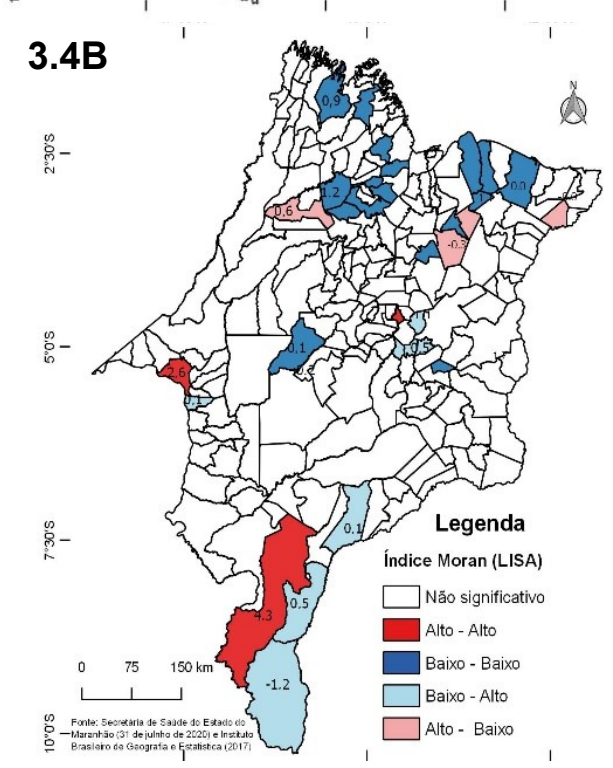

CASOS CONFIRMADOS DE COVID-19 E DESIGUALDADE SOCIAL

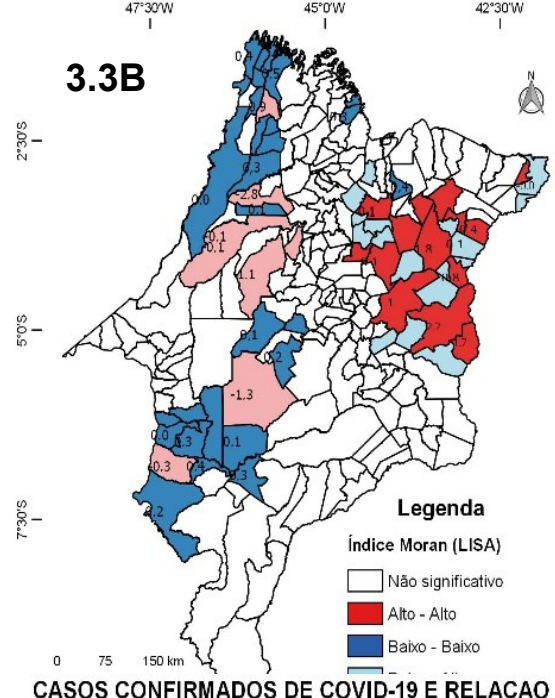

CASOS CONFIRMADOS DE COVID-19 E RELAÇAO

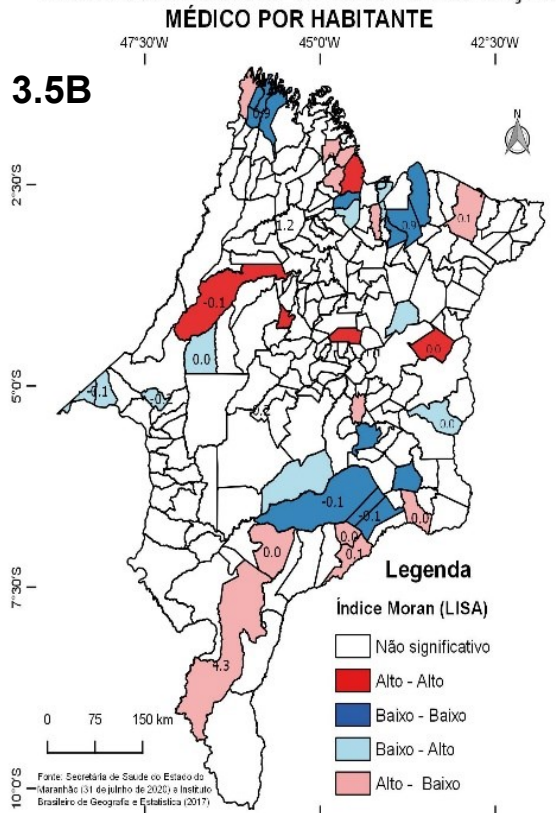




\section{Aspectos socioeconômicos e a Covid-19}

$\mathrm{O}$ índice de desenvolvimento humano municipal (IDHM) no Maranhão é baixo, conforme figura 3.2A, os municípios com esse indicador baixo foram: Fernando Falcão, Jenipapo dos Vieiras e Marajá do Sena. Nesses municípios a expectativa de vida é menor que 69 anos. No que diz respeito aos aspectos socioeconômicos, a oferta irregular ou desqualificada de recursos hídricos e de saneamento, deve acarretar a piora dos rendimentos médios por família e, por conseguinte, um comprometimento maior da renda mensal em busca de satisfazer-se dos recursos vitais fundamentais. Para os negócios e investimentos, mesmo que de pequenas e médias empresas familiares ou informais, a baixa qualidade na oferta dos serviços hídricos básicos, deve acarretar desestímulos, gerando o ciclo básico de prisão na baixa renda e dependência estatal (ROLNIK e KLINK, 2011).

O IDHm refletiu a desigualdade social da população maranhense (Figura 3.3A), dado que $72 \%$ (157) dos municípios, a desigualdade maior foi encontrada nos municípios de Santa Quitéria do Maranhão $(0,99)$, Afonso Cunha e Aldeias Altas, com 0,97. Os municípios que apresentaram classificação Alta Alta (vermelho), pelo Lisa, foram os localizados na parte Leste (Figura $3.3 \mathrm{~B}$ ), conforme os mapas de oferta de água, de saneamento e de qualidade dos serviços, tratam-se do agrupamento de municípios de maior insegurança hídrica e oferta água de qualidade.

A desigualdade social é um aspecto marcante no Maranhão, especialmente, na parte Leste do Estado. Nessa região, Silva, Santos e Vieira (2017), destacaram que nos municípios de Caxias, Codó e Timon, merecem atenção especial do poder público, devido às condições socioambientais e socioeconômicos. Trata-se da população sem tratamento adequado da água e saneamento, além de baixa segurança nos serviços prestados.

Para a desigualdade em relação aos casos confirmados, nota-se dois padrões específicos de comportamento. O primeiro, é confirmado no eixo de municípios de menor desigualdade que vai do Sul e Noroeste do estado, com índice "Baixo - Baixo" em azul, no mapa 3.3B. Para Moutinho (2020) a Covid-19 ao se instalar nas áreas mais pobres, onde falta água para lavar as mãos e onde as pessoas têm que se arriscar e descumprir o isolamento social para conseguir alguma renda para manter a sobrevivência de sua família. Conforme o mesmo autor, as desigualdades sociais se mostram como fatores determinantes no processo saúde-adoecimento da população brasileira. Já o segundo aspecto está relacionado à lentidão com que as áreas menos desenvolvidas apresentam em receber apoio estatal.

Apesar do maior PIB per capita do Maranhão que foi encontrado no município de São Antônio dos Lopes, com mais de $\mathrm{R} \$ 100.000,00$ (Figura 3.4 A), o índice de GINI ainda representa um elevado nível de desigualdade e o IDH permanece em 0,566 conforme a média do estado (2.5 A). Neste município, ainda, está localizado o Complexo Termelétrico Parnaíba, um dos maiores complexos de geração de energia a gás natural da América Latina, com 1,4 GW de capacidade instalada. É notável, portanto, que os maiores salários e rendimentos do complexo, não reflete diretamente em melhoria geral para a população, mas sim, para cargos e empregos muito específicos.

Algo semelhante ocorre com o município de Balsas que apresenta elevado PIB per capita, com baixo IDH (Figura 2.4 A). Para esse indicador a análise de correlação pelo LISA, demostrou categorias AltaAlta, sobretudo, para os municípios de Imperatriz e Balsa (Figura 2.4 B). O primeiro município estimulado pelos serviços, com PIB de $\mathrm{R} \$ 3.335 .822,3$ (x1000); e o segundo pelo agronegócio, em especial, a soja com área destinada em 157.657 hectares. Soma-se que, ao aplicar o Moran Global entre casos confirmados e PIB nos municípios, o resultado foi de -0.05 . Relativamente, a maior concentração de casos, pelo índice LISA, ocorre justamente nas áreas de maior renda e melhor segurança na oferta de recursos hídricos, mesmo que a baixa qualidade de vida e a desigualdade permaneçam consistentes nessas mesmas áreas.

\section{Saúde e a Covid-19}

A pandemia demostrou a deficiência na saúde no estado, no qual a maior concentração de médicos por mil habitantes foi de apenas 3,5 (Figura $3.5 \mathrm{~A}$ ). No Maranhão a média é de 0,8 médico por mil habitantes. A menor quantidade de médicos por mil habitantes foi encontrada em Conceição do Lago Açu $(0,1)$, Governador Newton Belo $(0,2)$ e Anapurus $(0,3)$. Para os casos confirmados de Covid-19 esses municípios tiveram 146, 403 e 646 casos, respetivamente. Ao aplicar o Moran Global entre casos confirmados e PIB nos municípios o resultado foi de - 0.05. Relacionando os dados, as áreas e agrupamentos com melhores índices de oferta de serviços é aquela que apresenta a maior

DOI:http://dx.doi.org/10.14393/Hygeia16057180 $\quad$ Hygeia $\quad$ v.16 $\quad$ p. $419-430, \quad 2020$ página 427


concentração de médicos por mil habitantes, contabilizando rede pública e privada. Para Silveira e Pinheiro (2014), os índices de médico por habitante são sempre mais favoráveis nas regiões economicamente mais privilegiadas, apontando uma relação estreita entre assistência médica e concentração de capital.

Para Lana et al (2020) a chegada do novo vírus colocou à prova a estrutura de vigilância existente no país, principalmente, com a redução de investimentos no Sistema Único de Saúde (SUS). O Brasil, já que foi protagonista na epidemia de Zika, que é transmitido pela picada do mosquito do gênero Aedes que se prolifera em locais sem saneamento básico (QUEIROZ; SILVA; HELLER, 2020).

Há necessidade de saúde preventiva e saneamento é essencial para as doenças gripais como a Covid19. Conforme relatos o vírus surgiu no final de 2019 na China, mas esse vírus já circula entre a população como encontrado por em Belém - PA, neste período os autores Nobre et al. (2014) recomendaram o monitoramento de sua circulação entre a população da região norte para que fosse erados dados epidemiológicos com o objetivo de diminuir os riscos de infecção por CoVh. O Sar-Cov2 (Covid-19) é um vírus que causa complicações respiratórias de leve a grave e elevada infectividade e patogenicidade, devido à sua alta transmissibilidade e falta de controle foi considerada uma pandemia pela Organização Mundial da Saúde (OMS).

A complexidade da incidência de casos de Covid-19 no estado do Maranhão se manifesta em diferentes escalas espaciais, impondo assim desafios analíticos $r$ para o planejamento de políticas públicas devido à falta de dados de qualidade ambiental e socioeconômicos.

\section{CONCLUSÃO}

O estudo demonstrou que a qualidade da água e a falta de coleta e tratamento de esgoto podem estar diretamente relacionadas à proliferação da Covid-19 entre a população maranhense, pois uma das medidas preventivas é a higiene. Como a debilidade do saneamento é notada em grande parte do estado, o grau de vulnerabilidade é ampliado para o caso estudado. Em relação aos aspectos socioeconômicos, notou-se que a maior influência da desigualdade, da falta de tratamento do saneamento básico e o baixo IDH são potenciais difusores da doença - não somente da Covid-19, mas dos desafios da saúde em geral. São os desafios em geral que somados à pandemia, agravam a condição cotidiana da população.

Os dados apresentados, contudo, também confirmam a influência global e irrestrita da doença, uma vez que os próprios municípios com melhores índices socioeconômicos foram os que apresentaram maiores índices de contaminação até o momento - o que não significa afirmar que as áreas mais pobres estejam sob menor risco da doença. Presume-se que se trata de cidades com menores integrações regionais e que permaneceram mais isoladas nos picos pandêmicos. Se o relativo isolamento destas cidades as tornou mais imunes à pandemia, não se pode dizer o mesmo para as soluções do baixo desenvolvimento local. É de extrema importância, aliás, a busca pela integração regional e as correlações sociais para a melhoria dos índices econômicos e sociais. Trata-se de um nítido paradoxo entre o isolamento da pandemia e o isolamento econômico.

Enquanto isso, continua havendo o menor número de médicos, verificável em áreas de maiores desigualdades, piores IDHm e piores índices na prestação e segurança dos serviços hídricos. Acreditamos que somente um largo investimento em saneamento, educação e progressão dos índices econômicos é que garantiriam melhores condições para o enfrentamento de situações de crises semelhantes à estas. Concluímos, neste sentido, que a análise das correlações espaciais pelo Índice de Moran se mostra como uma ferramenta útil para a identificação das relações nas áreas analisadas, suas lacunas sócio espaciais e de identificação de áreas de riscos.

\section{REFERÊNCIAS}

ALMEIDA, Caio Américo Pereira de. Análise geoespacial dos casos de dengue e sua relação com fatores socioambientais em Bayeux - PB. Hygeia, v. 13, n. 26, p. 71-86, 2017. https://doi.org/10.14393/Hygeia132606

ANA. Agência Nacional das Águas. Atlas do Esgoto. Disponível em: http://atlasesgotos.ana.gov.br/. Acesso em: 08 de julho de 2020.

DOI:http://dx.doi.org/10.14393/Hygeia16057180 $\quad$ Hygeia $\quad$ v.16 $\quad$ p. $419-430, \quad 2020$ página 428


ANA. Agência Nacional das Águas. Disponível em: https://metadados.ana.gov.br/geonetwork/srv/pt/main.home. Acesso em: 12 de agosto de 2020.

ANA. Agência Nacional das Águas. Plano Nacional de Segurança Hídrica. Disponível em: http://pnsh.ana.gov.br/. Acesso em: 09 de julho de 2020.

ARCHELA, Rosely Sampaio; THÉRY, Hervé. Orientação metodológica para construção e leitura de mapas temáticos. Confins, v. 3, 2008. https://doi.org/10.4000/confins.3483

BARCELLOS, Christovam; BUZAI, Gustavo D.; HANDSCHUMACHER, Pascal. Geografia e saúde: o que está em jogo? História, temas e desafios. Confins, v. 37, n. 37, p. 1- 11, 2018. https://doi.org/10.4000/confins.14954

BARCELLOS, Christovam; QUITÉRIO, Luiz Antônio Dias. Vigilância ambiental em saúde e sua implantação no Sistema Único de Saúde. Revista de Saúde pública, v. 40, p. 170-177, 2006. https://doi.org/10.1590/S0034-89102006000100025

CASANOVA, Lisa; RUTALA, William A.; WEBER, David J.; SOBSEY, Mark D. Survival of surrogate coronaviruses in water, Water Research, v. 43, p.1893-1898, 2009. https://doi.org/10.1016/j.watres.2009.02.002

CLARKE, Keith C.; MCLAFFERTY, Sara L.; TEMPALSKI, Barbara J. On Epidemiology and Geographic Information Systems: A Review and Discussion of Future Directions On Epidemiology and Geographic Information Systems: A Review and Discussion of Future Directions. Emerging Infectious Diseases, v. 2, n. 2, p.85-92, 1996. https://doi.org/10.3201/eid0202.960202

CPRM. Serviço Geológico do Brasil. Geodiversidade do Estado do Maranhão.Org. Iris Celeste Nascimento. Teresina-PI: CPRM, 2013. 294 p. (Programa Geologia do Brasil. Levantamento da Geodiversidade).

FERNANDES, Valdir; SAMPAIO, Carlos Alberto Cioce. Na environmental or a socio-environmental issue? The nature of the Society-environment relationship. Desenvolvimento e Meio Ambiental, $\mathrm{n}$. 18, p.87-94, 2008. https://doi.org/10.5380/dma.v18i0.13427

FUNASA. Fundação Nacional de Saúde. Impactos na saúde e no sistema único de saúde decorrentes de agravos relacionados a um saneamento ambiental inadequado. Brasília: Fundação Nacional de Saúde, 2010. $246 \quad$ p. Disponível em: http://www.funasa.gov.br/site/wpcontent/files_mf/estudosPesquisas_ImpactosSaude.pdf. Acesso em:08 nov.2020

GUIMARÃES, Raul Borges. Geografia e saúde coletiva no Brasil. Saúde Soc., v.25, n.4, p.869-879, 2016. https://doi.org/10.1590/s0104-12902016167769

IBGE. Maranhão. Disponível em: https://www.ibge.gov.br/cidades-e-estados/ma/.html?. Acesso em: 30 jul. 2019.

JUNQUEIRA, Renata Dias. Geografia médica e geografia da saúde. Hygeia, v. 5, n.8, p.57 - 91, 2009.

LANA, Raquel Martins; COELHO, Flávio Codeço; GOMES, Marcelo Ferreira da Costa; CRUZ, Oswaldo Gonçalves; BASTOS, Leonardo Soares; VILLELA, Daniel Antunes Maciel; CODEÇO, Cláudia Torres. Emergência do novo coronavírus (SARS-CoV-2) e o papel de uma vigilância nacional em saúde oportuna e efetiva. Cad. Saúde Pública, v. 36, n. 3, p. 1- 5, 2020. https://doi.org/10.1590/0102$\underline{311 \times 00019620}$

LUZARDO, Antônio José Rocha; CASTAÑEDA FILHO, Rafael March; Rubim, Igor Brum. Análise espacial exploratória com o emprego do índice de Moran. GEOgraphia, v.19, n. 40, p. 161 - 179, 2017. https://doi.org/10.22409/GEOgraphia2017.1940.a13807

MARANHÃO. Secretária de Saúde. Disponível em: https://painel-covid19.saude.ma.gov.br/. Acesso em: 31 de julho de 2020.

MELO, Marilia Carvalho de; JOHNSSON, Rosa Maria Formiga. O conceito emergente de segurança hídrica. Sustentare, v. 1, n. 1, p.72-92, 2017. https://doi.org/10.5892/st.v1i1.4325

MOUTINHO, Flavio Fernando Batista. Conflitos da sociedade brasileira com as normas sanitárias: um paralelo entre a revolta da vacina e a pandemia de Covid-19. Hygeia, Edição Especial: Covid-19, p.60 - 71, 2020. https://doi.org/10.14393/Hygeia0054392

DOI:http://dx.doi.org/10.14393/Hygeia16057180 $\quad$ Hygeia $\quad$ v.16 $\quad$ p. $419-430,2020$ página 429


NOBRE, Akim Felipe Santos; SOUSA, Rita Catarina Medeiros; SANTOS, Mirleide Cordeiro dos; BARBAGELATA, Luana Soares. Primeira detecção de coronavírus humano associado à infecção respiratória aguda na Região Norte do Brasil. Rev Pan-Amaz Saude, v.5, n.2, p. 37-41, 2014. https://doi.org/10.5123/S2176-62232014000200005

NUNES, Fabrizia Gioppo. Análise exploratória espacial de indicadores de desenvolvimento socioambiental das regiões de planejamento do Norte e Nordeste goiano. Ateliê Geográfico, v. 7, n. 1, p.237-259, 2013. https://doi.org/10.5216/ag.v7i1.19809

OMS. Água, saneamento, higiene e gestão de resíduos para o vírus da COVID-19. Disponível em: https://apps.who.int/iris/bitstream/handle/10665/331846/WHO-2019-nCoV-IPC_WASH-2020.3-por.pdf. Acesso em: 20 jul. 2020.

PELICIONI, Maria Cecília Focesi. Educação ambiental, qualidade de vida e sustentabilidade. Sociedade e Ambinete, v. 7, n.2, p. 19-31, 1998. https://doi.org/10.1590/S0104-12901998000200003

QUEIROZ, Josiane Teresinha Matos de; SILVA, Priscila Neves; HELLER, Léo. Novos pressupostos para o saneamento no controle de arboviroses no Brasil. Cad. Saúde Pública, v. 36, n.4, p.1-15. https://doi.org/10.1590/0102-311x00223719

RIGOTTO, Raquel Maria; AUGUSTO, Lia Giraldo da Silva. Saúde e ambiente no Brasil: desenvolvimento, território e iniqüidade social. Cad. Saúde Pública, v. 23 Sup 4, p.475-501, 2007.

RIOS, L. Geografia do Maranhão. $4^{a}$ edição revisada e atualizada. São Luís: Central dos Livros, 2005. 278p. https://doi.org/10.1590/S0102-311X2007001600002

ROLNIK, Raquel; KLINK, Jeroen. Crescimento econômico e desenvolvimento urbano: por que nossas cidades continuam tão precárias? Novos estud. - CEBRAP, n. 89, São Paulo, Mar. 2011. https://doi.org/10.1590/S0101-33002011000100006

SILVA, Aichely Rodrigues da; FONSECA, Alessandra Larissa D'Oliveira. Panorama da qualidade das águas brasileiras: uma abordagem do Estado do Maranhão. IN: SANTOS, Luiz Carlos Araújo dos; SEABRA, Geovanni de Farias; CASTRO, Claúdio Eduardo de. Geografia: trabalho, sociedade e meio ambiente. São Luís, Eduema, 2018.

SILVA, Aichely Rodrigues da; SANTOS, Rodrigo Lima; VIEIRA, Breno Thiago Salgado Valadares. Análise de indicadores socioeconômicos e ambientais utilizando o software R: averiguação da conjuntura de municípios maranhenses. Revista Percurso, v. 9, n. 1, p. 199- 217, 2017

SILVA, Gilmar Silvério da Silva; SANTOS, Edmilsom Arruda dos; CORREAA, Luciana Barbosa, et al. Avaliação integrada da qualidade de águas superficiais: grau de trofia e proteção da vida aquática nos rios Anil e Bacanga, São Luís (MA). Eng Sanit Ambient, v.19, n.3, 245-251, 2014. https://doi.org/10.1590/S1413-41522014019000000438

SILVEIRA, Rodrigo Pinheiro; PINHEIRO, Roseni. Entendendo a necessidade de médicos no interior da Amazônia - Brasil. Rev. bras. educ. med., v.38, n.4, p.451-459, 2014. https://doi.org/10.1590/S0100$\underline{55022014000400006}$

SOUZA, Nicolas Pereira de Souza; SILVA, Elisa Mousinho Gomes Carvalho Silva; TEIXEIRA, Marcelo Dias, et al. Aplicação do Estimador de Densidade kernel em Unidades de Conservação na Bacia do Rio São Francisco para análise de focos de desmatamento e focos de calor. Anais... XVI Simpósio Brasileiro de Sensoriamento Remoto - SBSR, Foz do Iguaçu, PR, Brasil, 13 a 18 de abril de 2013.

Trata Brasil. Painel do Saneamento. Disponível em: http://www.tratabrasil.org.br/comunicacao/painelsaneamento-brasil. Acesso em: 08 de julho, 2020.

ZHOU, Q. et al. Origin of SARS from accelerated evolution of a virus by combined pollution. The journal of applied ecology, v. 14, n. 8, p. 1374-1378, 2003. 